\title{
Probabilistic analysis of spills from stormwater detention facilities
}

\author{
G. Becciu \& A. Raimondi \\ Politecnico di Milano, Italy
}

\begin{abstract}
Stormwater detention facilities are important tools for flood control in modern urban drainage systems, since they reduce uncontrolled discharges into receiving water bodies and improve the efficiency of stormwater treatment plants. In their modelling it is important to limit the number and the amount of spills to ensure the maximum efficiency of the storage capacity. In this paper, the overflow probability distribution function from a stormwater detention facility is derived; the possibility of pre-filling of the storage capacity from previous runoff events, that can strongly influence spilled volumes, is considered. Effects of the control variables and of two different regulation rules on resulting expressions are investigated and discussed. Finally, an application to a case study is presented.

Keywords: probabilistic analysis, stormwater detention facility, spilled volume, pre-filling, management rule.
\end{abstract}

\section{Introduction}

In many urban areas, the significant and rapid increase of impervious surfaces, observed in the last decades, has made flood control mandatory. Stormwater detention facilities have proved to be an effective solution to this kind of problems both in separated and combined sewer systems [1, 2]. Their main function is to limit uncontrolled and polluted spills from drainage systems into receiving water bodies, improving water quality. Therefore, the modelling of stormwater detention facilities should focus on their capacity to store overloads from drainage systems and cope with spills.

Traditionally, their design is based on the so-called "design storm" approach, that assumes a "critical" rainfall event, extracted from a recorded series or defined by a standard pattern (rectangular, triangular, Chicago, etc.), as input in a 
rainfall-runoff model [3]. This critical rainfall event is considered isolated from the whole rainfall stochastic process and the storage capacity is always assumed empty at the beginning of runoff. Pre-filling from previous events is then neglected and an underestimation of the minimum storage volume can occur, strongly influencing its capacity of limiting uncontrolled spills into receivers.

Approaches based on continuous simulation are generally more reliable and lead to more accurate results, modelling the whole chain of recorded rainfalls events.

However, these methods are more complexes and their application can be difficult due to the lack of continuous long-term series of recorded rainfall events $[4,5]$.

As an alternative, in the nineties, some authors have proposed the application of an analytical probabilistic approach to the modelling of stormwater detention facilities [6-9]. The aim is to derive the Probability Distribution Functions (PDFs) of some characteristics of the storage process from those of the rainfall variables of interest and from the characteristics of the drainage catchment. By mean of some simplifying hypotheses, analytical probabilistic methods generally allow to model stormwater detention facilities, combining the simplicity of "design storm" methods and the probabilistic reliability of continuous simulations. Derived expressions are generally easy to implement and provide useful information to designers. In recent years, many applications and developments of this kind of approaches have been proposed in the literature [10, $11]$.

In particular, the authors [12-14], have deeply investigated the problem of pre-filling of the storage capacity from previous rainfall events and its relation with control variables and management rules of releases. Pre-filling volumes from previous rainfall events high increase the probability of spills into receiving waters, whose limitation is one of the main targets in the modelling of stormwater detention facilities.

In this paper, overflow PDFs have been derived, analysing two different strategies of runoff control and considering the possibility of pre-filling from a previous rainfall event. Effects of simplifying hypothesis and of control variables on results have been deeply investigated. Finally, the proposed approach has been applied to a case study in Milano, Italy, and results have been presented and discussed.

\section{Modelling of the storage process}

In the probabilistic modelling of the storage process, some hypotheses have been assumed to simplify the analytical derivations, as usual with this kind of approaches:

- on-line stormwater detention facilities have been considered: when the storage capacity is full and inflow occurs at a greater rate than outflow, the excess volume is spilled. 
- a couple of rainfall events have been considered; as discussed by [14], this assumption can be reliable only when a sufficiently long IETD and high outflow rates are considered. For stormwater detention facilities with low outflow rates (e.g., infiltration basins) or when strict limitations on discharges in the downstream drainage system are imposed, this hypothesis can underestimate the pre-filling probability and, as consequence, the overflow probability;

- incoming hydrographs have been assumed rectangular (constant runoff); this hypothesis can be acceptable since in the modelling of stormwater detention facilities, rainfall volumes are more influent than rainfall intensities. Moreover, the pattern of incoming hydrographs can be neglected, especially when inflow rates are higher than outflow rates;

- hydrological losses $(L)$ for infiltration, evapotranspiration and filling of depression storages have been averaged over whole rainfall duration;

- rainfall-runoff transformation has been neglected and net rainfall intensities have been considered as inflow rates in the basin. This hypothesis can be reliable only for small catchments; as a consequence, runoff duration is considered equal to rainfall duration, although the duration of a runoff event is usually longer than that of a rainfall event;

- outflow rates from the stormwater detention facility has been assumed constant. This is not easy to accomplish, since most of outlets have a linear logarithmic relationship between headwater depth and discharge. Also when the basin is emptied by a pump, the efficiency of the system can vary with the submergence of the pump, but in this case the outflow rate may be assumed constant.

To identify independent rainfall events from the continuous series of records, a minimum intervent time (IETD) has been defined; if the interevent time is lower than IETD, two consecutive rainfalls are joined together into a single event, otherwise they are considered independent.

The main characteristics of the rainfall process, rainfall depth $(h)$, rainfall duration $(\theta)$ and interevent time $(d)$ have been considered independent random variables exponential distributed:

$$
\begin{gathered}
f_{h}=\xi e^{-\xi h} \\
f_{\theta}=\lambda e^{-\lambda \theta} \\
f_{d}=\psi e^{-\psi(d-I E T D)}
\end{gathered}
$$

where: $\xi=1 / \mu_{h}, \lambda=1 / \mu_{\theta}$ and $\psi=1 /\left(\mu_{d}-\right.$ IETD $) ; \mu_{h}$ : average rainfall depth, $\mu_{\theta}$ : average rainfall duration, $\mu_{d}$ : average interevent time. 
Although other PDF seem to be more correct to fit experimental data $[5,14]$ this hypothesis is often considered acceptable in order to reduce the complexity of analytical derivation [15-20].

Two different management rules of the stormwater detention facility have been analysed according to the more frequent strategies of runoff control.

Management rule A: the stormwater detention facility is emptied, with a constant outflow rate $(q)$, starting as soon as it begins to fill. Considering rectangular events with incoming runoff rates greater than outflow rates, this means soon after the beginning of each event (Figure 1). Management rule A is typical of on-line stormwater detention facilities.

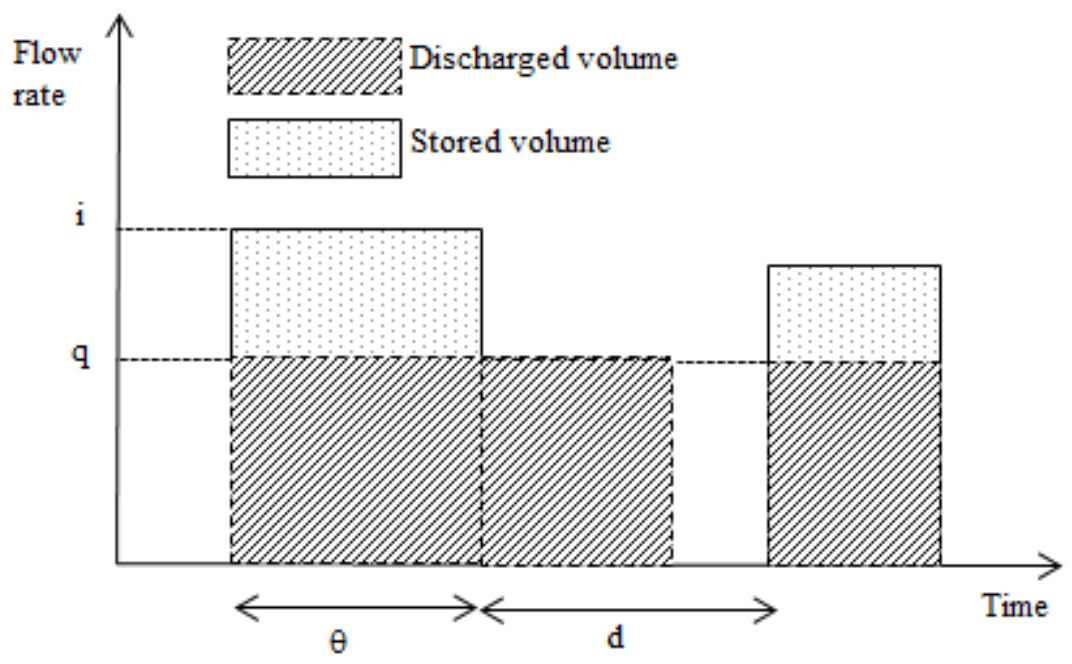

Figure 1: Management rule A.

Management rule B: the stormwater detention facility is emptied, with a constant outflow rate $(q)$, only after the end of each runoff event. The outflow goes on until the facility is empty or a next event begins (Figure 2). Management rule B can be used in Real Time Control (RTC) applications, when is necessary to temporarily retain a certain volume to reduce the risk of downstream system overload, or for water quality control purposes.

\section{Probability distribution functions of spilled volumes}

The overflow PDF has been derived with reference to the above described management rules. In particular, the probability that spilled volume $\left(w_{s}\right)$ exceeds a fixed percentage $(\alpha)$ of storage capacity $\left(w_{0}\right)$ has been estimated; it has been denoted by $P$, that is: $P=P\left(w_{s}>\alpha \cdot w_{0}\right)$.

Considering a couple of consecutive runoff events, conditions for spills are:

- $\quad$ spill of the first runoff event only; 
- $\quad$ spill of both runoff events with pre-filling from the first event at the beginning of the second one;

- $\quad$ spill of both runoff events without pre-filling;

- $\quad$ spill of the second runoff event only, with pre-filling from the first event at the beginning of the second one;

- $\quad$ spill of the second runoff event only, without pre-filling.

Under the hypothesis that $h=h_{1}=h_{2}, \theta=\theta_{1}=\theta_{2}$ and $d=d_{1}=d_{2}$, the first and the last conditions can't occur.

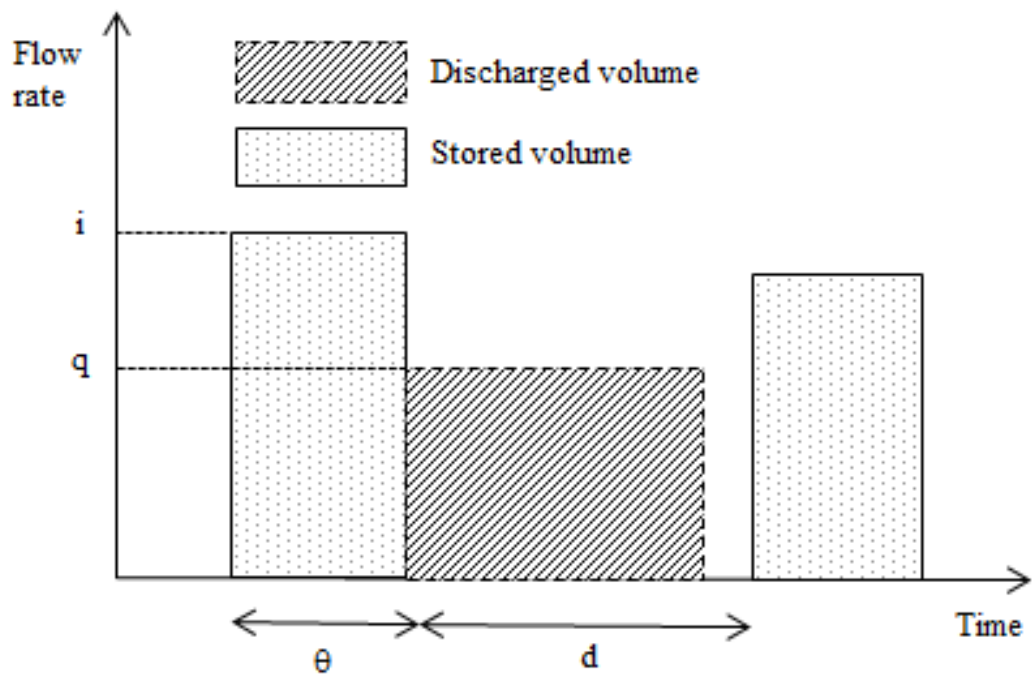

Figure 2: Management rule B.

In all formulas, volumes and flow rates have been expressed per unit of effective catchment area $(\varphi \cdot S$, where $\varphi$ is the runoff coefficient and $S$ is the catchment area). Furthermore, the following auxiliary dimensionless variables have been used:

$$
\beta=\frac{q \cdot \xi}{q \cdot \xi+\psi}, \beta^{*}=\frac{q \cdot \xi}{q \cdot \xi+2 \cdot \psi}
$$

and $q^{*}=q \cdot \xi / \lambda$.

Management rule A

Spilled volume can be expressed by:

$$
w_{s}=\left\{\begin{array}{lr}
2 \cdot(h-L-q \cdot \theta)-q \cdot d-w_{0} & \text { Case I } ; \text { Case II } \\
2 \cdot\left(h-L-q \cdot \theta-w_{0}\right) & \text { Case III } \\
0 & \text { Otherwise }
\end{array}\right.
$$


Case I:

$h-L-q \cdot \theta-w_{0}>0$;

$w_{0}-q \cdot d>0$;

$2 \cdot(h-L-q \cdot \theta)-q \cdot d-w_{0}>0$

Case II:

$h-L-q \cdot \theta-w_{0} \leq 0$;

$h-L-q \cdot(\theta+d)>0$;

$2 \cdot(h-L-q \cdot \theta)-q \cdot d-w_{0}>0$

Case III:

$h-L-q \cdot \theta-w_{0}>0$;

$w_{0}-q \cdot d \leq 0$;

$2 \cdot\left(h-L-q \cdot \theta-w_{0}\right)>0$

The overflow PDF can be estimated combining conditions of equation (4) together and using the PDFs of rainfall depth (1), duration (2) and interevent time (3).

If $w_{0} / q>I E T D$, that is considering the possibility of pre-filling from the first runoff event at the beginning of the second one, it results:

$$
P_{A}=\int_{\theta_{A}}^{\theta_{B}} f_{\theta} \cdot d \theta\left(\int_{d_{A}}^{d_{B}} f_{d} \cdot d d \int_{h_{A}}^{h_{B}} f_{h} \cdot d h+\int_{d_{A}}^{d_{B}} f_{d} \cdot d d \int_{h_{A}}^{h_{B}} f_{h} \cdot d h\right)
$$

where:

$\theta_{A}=0 ; \theta_{B}=\infty$

$d_{A}=I E T D ; d_{B}=d_{C}=w_{0} / q ; d_{D}=\infty$

$h_{A}=L+\left[w_{0}(1+\alpha)+q \cdot d\right] / 2+q \cdot \theta ; h_{B}=h_{D}=\infty ; h_{C}=L+w_{0}+q \cdot \theta+$ $\alpha \cdot w_{0} / 2$

Solving equation (5), the following expression can be obtained:

$$
P_{A}=\frac{e^{-\xi \cdot L}}{1+q^{*}}\left\{\left(1-\beta^{*}\right) e^{-\frac{\xi}{2}\left[q \cdot I E T D+w_{0}(1+\alpha)\right]}+\beta^{*} \cdot e^{\psi \cdot I E T D-\xi \cdot w_{0}\left(\frac{\alpha}{2}+\frac{1}{\beta}\right)}\right\}
$$

If $w_{0} / q \leq I E T D$, pre-filling from the first runoff event can't occur and the overflow PDF results:

where:

$$
P_{A}=\int_{\theta_{A}}^{\theta_{B}} f_{\theta} \cdot d \theta \int_{d_{A}}^{d_{B}} f_{d} \cdot d d \int_{h_{A}}^{h_{B}} f_{h} \cdot d h=\frac{1}{1+q^{*}} \cdot e^{-\xi \cdot\left[L+w_{0}\left(1+\frac{\alpha}{2}\right)\right]}
$$

$\theta_{A}=0 ; \theta_{B}=\infty$

$d_{A}=I E T D ; d_{B}=\infty$

$h_{A}=w_{0}+q \cdot \theta+\alpha \cdot w_{0} / 2+L ; h_{B}=\infty$ 


\section{Management rule B}

Spilled volume can be expressed by:

Case I:

$$
w_{s}=\left\{\begin{array}{lr}
2 \cdot(h-L)-q \cdot d-w_{0} & \text { Case I } ; \text { Case II } \\
2 \cdot\left(h-L-w_{0}\right) & \text { Case III } \\
0 & \text { Otherwise }
\end{array}\right.
$$

$h-L-w_{0}>0$;

$w_{0}-q \cdot d>0$

$2 \cdot(h-L)-q \cdot d-w_{0}>0$

Case II:

$h-L-w_{0} \leq 0$;

$h-L-q \cdot d>0$;

$2 \cdot(h-L)-q \cdot d-w_{0}>0$

Case III:

$h-L-w_{0}>0$;

$w_{0}-q \cdot d \leq 0$;

$2 \cdot\left(h-L-w_{0}\right)>0$

If $w_{0} / q>I E T D$, the overflow PDF results:

$$
P_{B}=\int_{d_{A}}^{d_{B}} f_{d} \cdot d d \int_{h_{A}}^{h_{B}} f_{h} \cdot d h+\int_{d_{A}}^{d_{B}} f_{d} \cdot d d \int_{h_{A}}^{h_{B}} f_{h} \cdot d h
$$

where:

$$
\begin{aligned}
& d_{A}=I E T D ; d_{B}=d_{C}=w_{0} / q ; d_{D}=\infty \\
& h_{A}=L+\left[w_{0}(1+\alpha)+q \cdot d\right] / 2 ; h_{B}=h_{D}=\infty ; h_{C}=L+w_{0}+\alpha \cdot w_{0} / 2
\end{aligned}
$$

Solving equation (9), the following expression can be obtained:

$$
P_{B}=e^{-\xi \cdot L} \cdot\left[\left(1-\beta^{*}\right) e^{-\frac{\xi}{2}\left[q \cdot I E T D+w_{0}(1+\alpha)\right]}+\beta^{*} \cdot e^{\psi \cdot I E T D-\xi \cdot w_{0}\left(\frac{\alpha}{2}+\frac{1}{\beta}\right)}\right]
$$

If $w_{0} / q \leq \operatorname{IETD}$, the overflow PDF results:

$$
P_{B}=\int_{d_{A}}^{d_{B}} f_{d} \cdot d d \int_{h_{A}}^{h_{B}} f_{h} \cdot d h=e^{-\xi \cdot\left[L+w_{0}\left(1+\frac{\alpha}{2}\right)\right]}
$$

where:

$d_{A}=I E T D ; d_{B}=\infty$

$h_{A}=w_{0}+\alpha \cdot w_{0} / 2+L ; h_{B}=\infty$

Results from management rules $\mathrm{A}$ and $\mathrm{B}$ are comparable, except for the independence of strategy control B of rainfall duration. Comparing equation (6) and (7) respectively with equation (10) and (11), it results:

$$
P_{A}=\frac{P_{B}}{1+q^{*}}
$$

As expected, overflow probability is lower with rule A than with rule B. 


\section{Case study}

Derived expressions for the estimation of overflow PDF have been applied to rainfall series recorded at gauge station of Milano-Monviso (Italy) during the period 1991-2005. With an IETD $=1$ hours, $N=1647$ rainfall events have been identified from the continuous chain of records. The values of parameters for the exponential PDF of considered rainfall variables are:

\begin{tabular}{cc}
\hline$\xi[1 / \mathrm{mm}]$ & 0,13 \\
$\lambda[1 /$ hour $]$ & 0,23 \\
$\psi[1 /$ hour $]$ & 0,01 \\
\hline
\end{tabular}

Hydrological losses have been set to $L=2 \mathrm{~mm}$. Average rainfall intensity is equal to $i=1,79 \mathrm{~mm} /$ hour. To maintain the constant outflow rate less than the average rainfall intensity, at first $q=1 \mathrm{~mm} /$ hour has been assumed.

Figure 3 compares the overflow PDF for management rule A and B, varying storage volume, for $\alpha=0$. This mean that only the probability to have overflows different from zero is estimated without information about the amount of spilled water. Obviously, rule B results more critical and leads to higher probabilities of overflow.

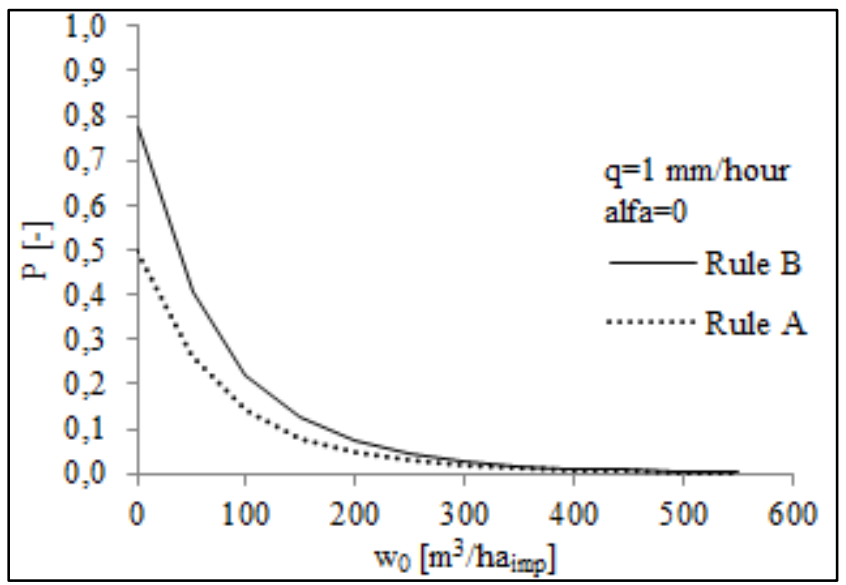

Figure 3: Overflow probability vs. storage volume and management rule.

With reference to management rule B only, Figure 4 compares the overflow PDF for the two boundary conditions of $\alpha$ coefficient, that is the probability of spilled volumes different from zero $(\alpha=0)$ and the probability of spilled volumes equal to storage capacity $(\alpha=1)$. 


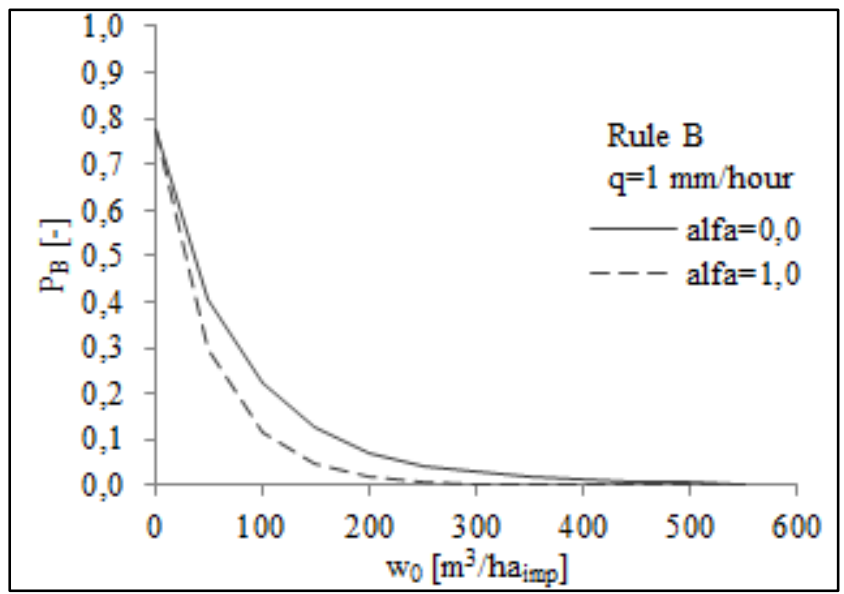

Figure 4: Overflow probability vs. storage volume and alfa.

For example, considering a storage capacity of $w_{0}=100 \mathrm{~m}^{3} / \mathrm{ha} \mathrm{imp}_{\text {, }}$, the probability of overflow is about $30 \%$, while the probability that $w_{s}=w_{0}=$ $100 \mathrm{~m}^{3} / \mathrm{ha} a_{\text {imp }}$ is about $10 \%$.

Always considering management rule $\mathrm{B}$, the influence of outflow rates on overflow probability is negligible for $q \geq 1 \mathrm{~mm} / \mathrm{hour}$ and is maximum for $q \rightarrow 0$ (Figure 5). This because, when outflow rate increases, the probability of pre-filling from previous rainfall events falls to zero; as consequence, a single rainfall event is considered for the estimation of the overflow PDF, that in this case, results independent of outflow rate.

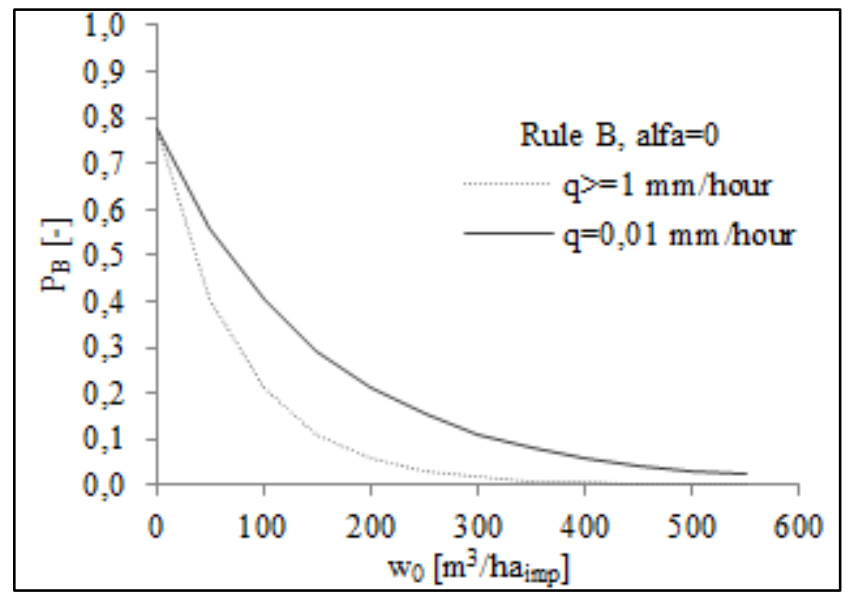

Figure 5: Overflow probability vs. storage volume and outflow rate. 
Otherwise, considering management rule $\mathrm{A}$, the overflow PDF is strongly influenced by outflow rates and it tends to zero, increasing outflow rate, already for small storage volumes (Figure 6); this because, with this strategy control of releases, the storage capacity starts emptying at the beginning of the rainfall event and, if the outflow rate is higher than rainfall intensity, stored volume tends to zero.

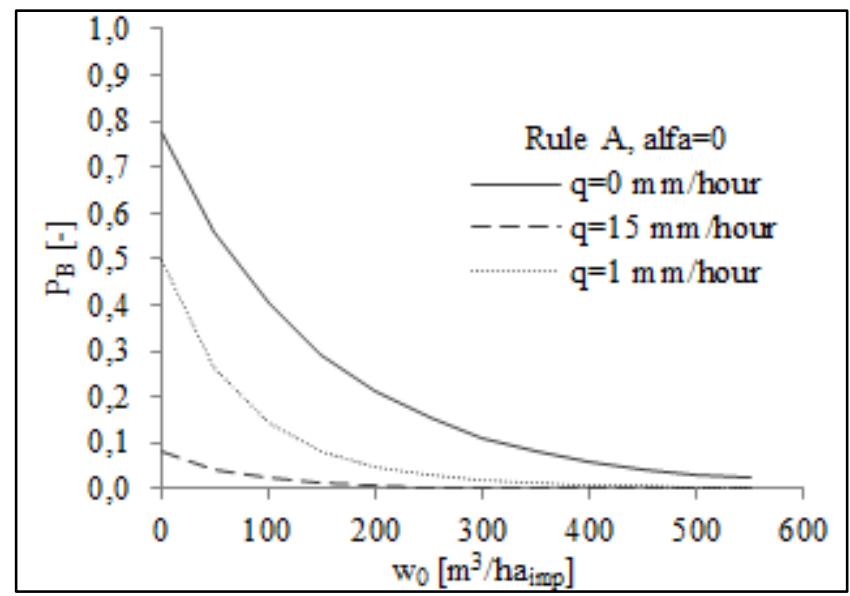

Figure 6: Overflow probability vs. storage volume and outflow rate.

Management rule $\mathrm{A}$ is preferable than management rule $\mathrm{B}$, when possible, to limit spills from stormwater detention facilities.

\section{Conclusion}

Stormwater detention facilities are important tools for flood control in urban drainage systems, because they increase the efficiency of treatment plants and improve the quality of discharges. For their proper modelling, uncontrolled spills into receiving water bodies should be avoid or kept to a minimum.

In this paper, an analytical probabilistic approach to estimate the overflow PDF has been proposed. A couple of rainfall events have been considered to take into account the possibility of pre-filling from a previous rainfall event that could increase the number and the amount of overflows. Two different management rules, in accordance with the control strategies of releases most used in practice, have been proposed and assumed as boundary conditions for the estimation of the PDFs of spilled volumes.

Derived expressions result function of the stochastic rainfall process, storage volume and outflow rate; they allow to estimate not only the overflow probability but also the probability to have a certain amount of spilled volume, related to the storage capacity. Moreover, minimum interevent time (IETD) and hydrological losses $(L)$ have been considered in resulting formulas. In the case study a minimum interevent time equal to $\operatorname{IETD}=1$ hour has been used; 
theoretically, in these conditions results could be affected by pre-filling from more than one previous event. However, for the observed data, with $q>$ $1 \mathrm{~mm} /$ hour, storage capacity results empty at the beginning of a new runoff and the simplifying assumption of considering only a couple of rainfall events can be considered acceptable.

Proposed formulas can be a valuable aid in the design of stormwater detention facilities, providing information about the probability and the amount of spilled volumes under different conditions. They can be suitable in many engineering applications, especially in the case of strict discharge limits or of infiltration basins with low permeability soils, and when the continuous simulation of series of recorded storm events is not possible or reliable according to the amount and quality of rainfall records.

\section{References}

[1] Urbonas B. and Stahre P., Stormwater: best management practices and detention for water quality, drainage and CSO management. PTR Prentice Hall, Englewood Cliffs, New Jersey, USA, 1993.

[2] ASCE, WEF, Urban runoff quality management, American Society of Civil Engineers, Reston, USA, 1998.

[3] Dooge, J.C.I., Problems and methods of rainfall runoff modeling, in: mathematical models for surface water hydrology. Ciriani T.A., Maione U., Wallis J.R. (Eds), John Wiley, NY, 71-108, 1977.

[4] Adams, B.J., Fraser, H.G., Howard C.D.D., and Hanafy, M.S., Meteorologic data analysis for drainage system design. Journal of Environmental Engineering, ASCE, Vol. 112, No. 5, 827-848, 1986.

[5] Bacchi B., Balistrocchi M. and Grossi G., Proposal of a semi probabilistic approach for storage facility design. Urban Water Journal Vol. 5, No 3, 195-208, 2008.

[6] Guo Y. and Adams B. J., Hydrologic analysis of urban catchments with event-based probabilistic models. 1. Runoff volume. Water Resources Research 34 (12), 3421-3443, 1998a.

[7] Guo Y. and Adams B. J., Hydrologic analysis of urban catchments with event-based probabilistic models. 2. Peak discharge rate. Water Resources Research 34 (12), 3421-3443, 1998 b.

[8] Guo Y. and Adams B. J., An analytical probabilistic approach to sizing flood control detention facilities. Water Resources Research 53 (8), 24572468, 1999.

[9] Adams, B.J. and Papa, F., Urban Stormwater Management Planning with analytical probabilistic models. John Wiley \& Sons, New York, USA, 1999.

[10] Guo Y. and Dai J., Expanded analytical probabilistic stormwater models for use in watershed and master drainage planning. Canadian Journal of Civil Engineering, 2009. 
[11] Guo Y. and Zhuge Z., Analytical probabilistic flood routing for urban stormwater management purposes. Canadian Journal of Civil Engineering, 2008.

[12] Becciu G., Paoletti A., Raimondi A., Sanfilippo U., Effects of management rules on pre-filling of stormwater detention facilities. 12th International Conference on Urban Drainage, Porto Alegre/Brazil, 2011.

[13] Becciu G., Raimondi A., Factors affecting the pre-filling probability of water storage tanks, WIT Transactions: Ecology and the Environment volume 164 (Water Pollution XI), 12 pp, WIT, U.K., 2012.

[14] Raimondi A., Becciu G., On pre-filling probability of flood control detention facilities, Urban Water Journal accepted for publication, 2014.

[15] Eagleson, P.S., Dynamics of flood frequency. Water Resources Research 8(4), 878-898, 1972.

[16] Eagleson, P.S., Climate, soil and vegetation, 2, The distribution of annual precipitation derived, 1978.

[17] Howard, C.D.D., Theory of storage and treatment plant overflows. Journal of Environmental Engineering, 102(EE4), 709-722, 1976.

[18] Adams, B.J. and Bontje, J.B., Microcomputer applications of analytical models for urban stormwater management. Emerging Computer Techniques in Stormwater and Flood Management, W. James, 138-162, Am. Soc. Civ. Eng., New York, 1984.

[19] Adams, B.J., Fraser, H.G., Howard C.D.D., and Hanafy, M.S., Meteorologic data analysis for drainage system design. Journal of Environmental Engineering, ASCE, Vol. 112, No. 5, 827-848, 1986.

[20] Bedient P. B. and Huber W.C., Hydrology and floodplain analysis, 2nd Ed., Addison Wesley, New York, USA, 1992. 